\title{
Kinetics of ion-beam damage in lithium niobate
}

\author{
A. García-Navarro and F. Agulló-López ${ }^{\text {a) }}$ \\ Centro de Microanálisis de Materiales (CMAM), Universidad Autónoma de Madrid (UAM), \\ Cantoblanco, E-28049 Madrid, Spain
}

M. Bianconi

CNR-IMM-Sezione di Bologna, Via P. Gobetti 101, I-40129 Bologna, Italy

J. Olivares ${ }^{\text {b) }}$

Instituto de Óptica, CSIC, C/Serrano 121, E-28006 Madrid, Spain

G. García

CELLS, E-08193 Bellaterra, Barcelona, Spain

(Received 22 November 2006; accepted 26 January 2007; published online 17 April 2007)

\begin{abstract}
The damage kinetics induced by irradiation with a diversity of swift ions $(\mathrm{O}$ at $5 \mathrm{MeV}$; $\mathrm{F}$ at 5.1 MeV; Si at 5, 7.5, and $41 \mathrm{MeV}$; and $\mathrm{Cl}$ at 11 and $46 \mathrm{MeV}$ ) has been investigated in the range of $10^{12}-10^{15}$ at. $/ \mathrm{cm}^{2}$. It covers from the initial stage where single damage tracks are isolated and well separated, up to the stage where a full amorphous layer is produced. The damage is characterized by the areal fraction of disorder derived from the Rutherford backscattering/ channeling spectra. The data approximately fit an abrupt Avrami-type dependence with fluence. The fluence value at which 50\% of the sample surface becomes disordered shows a clear increasing trend with the electronic stopping power of the ion. The trend is consistent with Monte Carlo simulations based on a recent model for defect creation. Moreover, the quantitative agreement for the defect generation rate appears also reasonable. (C) 2007 American Institute of Physics.
\end{abstract}

[DOI: $10.1063 / 1.2714772$ ]

\section{INTRODUCTION}

Ion irradiation and implantation have been extensively used to modify materials and improve their performance for specific device applications. ${ }^{1,2}$ In particular, for dielectric materials optical waveguides and integrated optic devices has been produced by ion implantation of light ions with energies in the range up to a few $\mathrm{MeV}$. In this case the damage is mostly caused by nuclear collisions and it is reasonably well understood. The situation appears more complicated in the case of heavier ions and higher energies, which are being increasingly used by researchers. In this case the electronic stopping power $\left(S_{e}\right)$ becomes dominant over that associated with nuclear collisions $\left(S_{n}\right)$. Useful data on such electronic excitation processes have been obtained in experiments, where the effect of single ion impacts has been investigated through the formation of nanometer size amorphous (latent) tracks along their trajectories. ${ }^{3-6}$ They reveal an important characteristic of electronic damage, namely, thresholding i.e., the sharp transition from the preamorphous (point-defect-like) to the amorphous stage above a certain threshold value of the electronic stopping power, which depends on the ion velocity. ${ }^{6}$ So far, thermal spike models have been mostly used to theoretically describe the main trends of amorphization. ${ }^{7-10}$ It is proposed that the deposited energy by the ion impact causes crystal melting that after rapid thermal quenching leads to amorphization.

The information is scarcer when the impact density or

\footnotetext{
a) Also at Departamento de Física de Materiales, Universidad Autónoma de Madrid (UAM), Cantoblanco, 28049 Madrid, Spain.

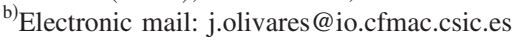

fluence increases and the single-impact processes interact and become more complicated. At high enough fluences where an average description is adequate and essentially homogeneous defective layers are formed, a number of Rutherford backscattering/channeling (RBS/C) spectra and optical results have been published. ${ }^{11-16}$ They have both confirmed the thresholding nature of the defect generation processes and have clearly stood out the cumulative character of the damage. Based on these results, the thermal spike concept has been extended ${ }^{17}$ to analytically describe the generation and growth of the amorphous regions with increasing fluence. The model has been satisfactorily applied to explain experimental data on $\mathrm{LiNbO}_{3}$ using Si-beam irradiations. ${ }^{15}$

On the other hand, experiments at low enough fluences appear quite relevant to investigate in detail the initial stages of damage where statistical fluctuations are relevant. This would allow as to clarify the defect-accumulation mechanisms and the coupling between damaged regions. In particular, for stopping powers below the threshold value one would monitor the processes going from the initial disperse regions containing point defects to the stage where overlapping is relevant and defect accumulation takes place. This finally leads to overcoming the threshold defect concentration causing lattice collapse and amorphization. Diverse experiments have provided kinetic descriptions for the RBS/C data in ion-beam irradiated $\mathrm{LiNbO}_{3}$ showing Avrami's-type dependences or related descriptions. ${ }^{6,12}$ On the other hand, a Monte Carlo simulation approach based on the thermal spike model has been developed ${ }^{18}$ to cope with the initial stage of damage, taking into account the role of statistical fluctuations. As a general rule, nuclear collision processes play a minor role 
TABLE I. Parameters for the irradiations and RBS/C analyses.

\begin{tabular}{|c|c|c|c|c|}
\hline Ion & Cut & $\begin{array}{l}\text { Energy } \\
(\mathrm{MeV})\end{array}$ & $\begin{array}{c}\text { Fluences } \\
\left(10^{13} \text { at. } / \mathrm{cm}^{2}\right)\end{array}$ & $\mathrm{RBS} / \mathrm{C}$ \\
\hline $\mathrm{O}$ & $X$ & 5.0 & $20,40,60$ & H $2.2 \mathrm{MeV}$ \\
\hline F & $X$ & 5.1 & $\begin{array}{c}0.1,0.5,1,2,4,10 \\
20,40,100,300\end{array}$ & $\mathrm{H} 3 \mathrm{MeV}$ \\
\hline $\mathrm{Si}$ & $\mathrm{Z}$ & 5.0 & $\begin{array}{c}0.2,0.4,1,2,4,10 \\
20,40,80,200\end{array}$ & $\mathrm{H} 3 \mathrm{MeV}$ \\
\hline $\mathrm{Si}$ & $\mathrm{Z}$ & 7.5 & $\begin{array}{c}0.1,0.2,0.4,0.8,1,2 \\
\quad 4,10,20,40,80\end{array}$ & $\mathrm{H} 3 \mathrm{MeV}$ \\
\hline $\mathrm{Si}$ & $X$ & 41 & $\begin{array}{c}0.1,0.2,0.4,1,1.5,2 \\
2.5,3,3.5,4,4.5,5\end{array}$ & $\mathrm{H} 3.8 \mathrm{MeV}$ \\
\hline $\mathrm{Cl}$ & $\mathrm{X}$ & 11 & $\begin{array}{c}0.08,0.1,0.2,0.4,0.8 \\
1,2,4,20\end{array}$ & H $2.2 \mathrm{MeV}$ \\
\hline $\mathrm{Cl}$ & $\mathrm{X}$ & 46 & $0.2,0.4,0.8,1$ & H $3 \mathrm{MeV}$ \\
\hline
\end{tabular}

under high-energy irradiation conditions, although some kinds of synergy between electronic and nuclear mechanisms should be very likely considered for a full description.

The purpose of this work is to study the kinetics of damage in $\mathrm{LiNbO}_{3}$ under a variety of irradiation conditions (ion type, energy, and fluence) in order to identify the defectaccumulation law, clarify the underlying physical mechanisms, and stand out the transition from the preamorphous to amorphous stage. $\mathrm{LiNbO}_{3}$ is a reference material for electrooptic and nonlinear optical applications and is used as a substrate for a variety of waveguide and integrated optic devices. ${ }^{19,20}$ A main objective of the work is to assess the role of the electronic stopping power on such kinetic behavior. Since the experimental information has been obtained by the RBS/C technique, the data do not directly give the defect concentrations or the extension of disordered areas. Therefore, one has to derive such information through a suitable scattering function that relates the $\mathrm{RBS} / \mathrm{C}$ response to the defect concentration. ${ }^{21}$ Then, one obtains the evolution of defect concentration with the irradiation fluence. The results are finally analyzed within a very general context, and the validity of the various theoretical approaches is comparatively assessed. A main conclusion is that the defect concentration is a smooth function of fluence up to a critical value where lattice collapse occurs, whereas the defect scattering function is a sharp function of concentration. The combination of these two dependences is responsible for the observed thresholding behavior.

\section{EXPERIMENT}

Crystal plates of congruent $\mathrm{LiNbO}_{3}$ purchased from CASIX were irradiated with $\mathrm{O}, \mathrm{F}, \mathrm{Si}$, and $\mathrm{Cl}$ ions at the 5 MV Tandetron accelerator recently installed at the CMAM in the Universidad Autónoma de Madrid. The irradiation parameters are summarized in Table I. The current density was lower than $200 \mathrm{nA} / \mathrm{cm}^{2}$ to minimize charging and heating. The samples were tilted $8^{\circ}$ relative to normal incidence in order to avoid channeling effects. The electronic stopping

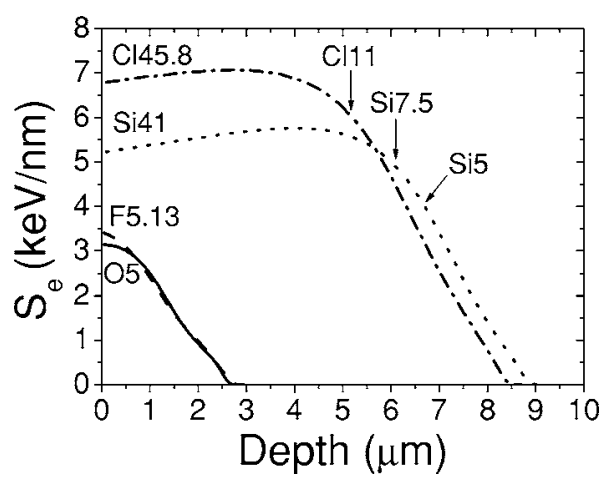

FIG. 1. Electronic stopping power curves for ions used in this work

power curves for all cases, derived from the SRIM code, ${ }^{22}$ are shown in Fig. 1.

$\mathrm{RBS} / \mathrm{C}$ spectra for the previously irradiated samples were taken along the $c$ axis in the IBM geometry, with the detector placed at $170^{\circ}$ (scattering angle) using $\mathrm{H}$ ions with energies also listed in Table I. The damage depth profiles were extracted from the $\mathrm{Nb}$ signal by using a program developed at IMM. $^{23}$ It is assumed that the defects generated by either nuclear or electronic mechanisms give the same RBS/channeling response.

\section{EXPERIMENTAL RESULTS ON DAMAGE KINETICS}

As an example, Fig. 2(a) illustrates the RBS/channeling spectra for samples irradiated with $\mathrm{F}$ ions $(5.1 \mathrm{MeV})$ at several fluences. The damage depth profiles derived from those raw data are displayed in Fig. 2(b). They represent the frac-
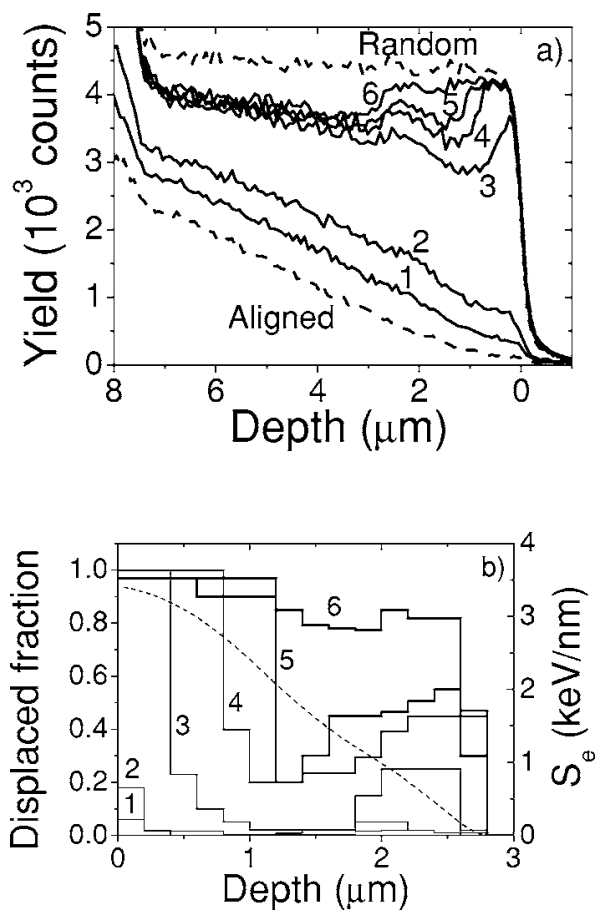

FIG. 2. (a) Relative RBS/C yield as a function of depth for samples irradiated with $\mathrm{F}$ (at $5.1 \mathrm{MeV}$ ) at several fluences: (1) $2 \times 10^{13} \mathrm{~cm}^{-2}$, (2) 4 $\times 10^{13} \mathrm{~cm}^{-2}$, (3) $1 \times 10^{14} \mathrm{~cm}^{-2}$, (4) $2 \times 10^{14} \mathrm{~cm}^{-2}$, (5) $4 \times 10^{14} \mathrm{~cm}^{-2}$, and (6) $1 \times 10^{15} \mathrm{~cm}^{-2}$. (b) Displaced atomic fraction as a function of depth derived from (a). The dashed line stands for the electronic stopping curve in the scale of the right hand side ordinate. 

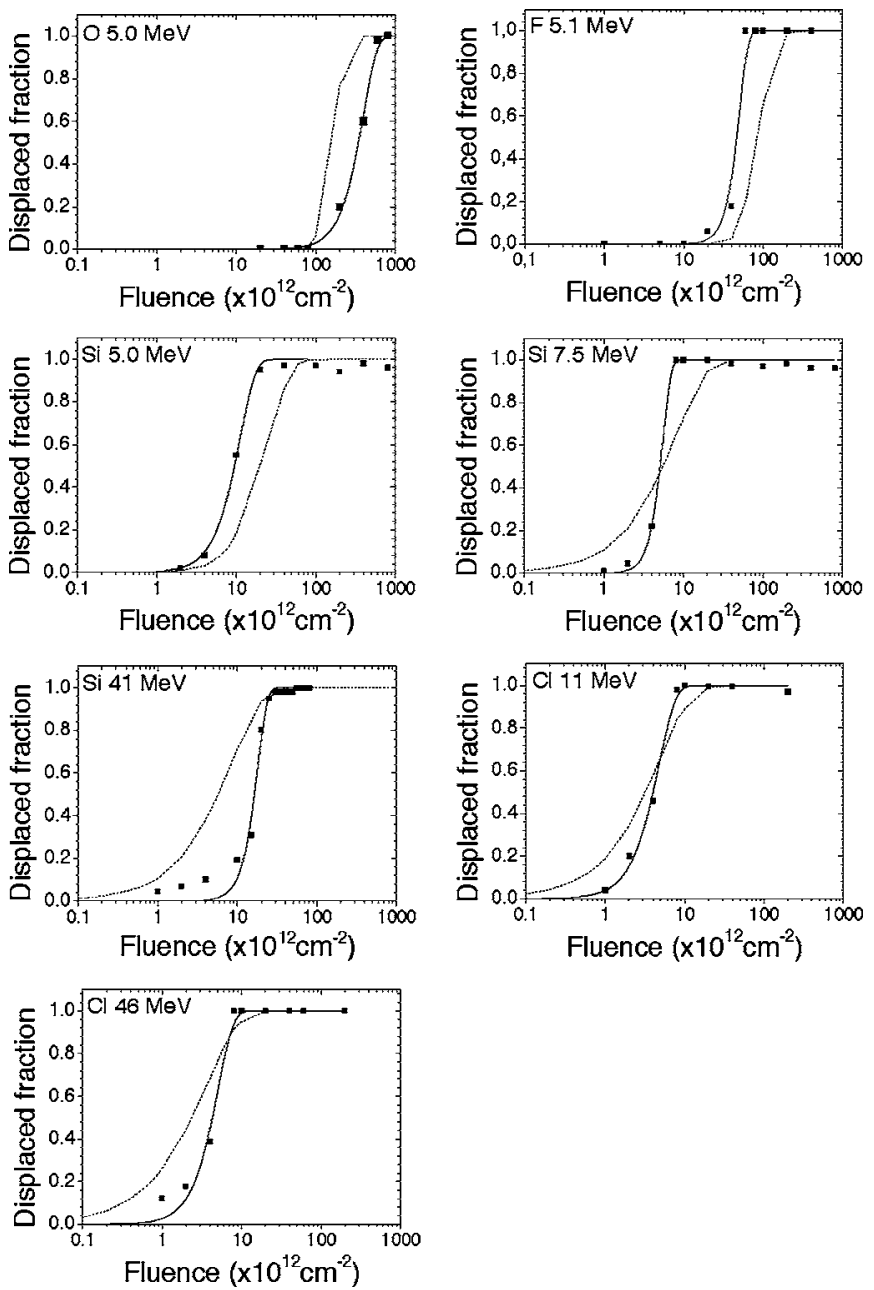

FIG. 3. Displaced fraction vs fluence for all investigated cases, Avrami's fits (continuous lines) and Monte Carlo fits (dashed lines).

tion $(s)$ of randomly displaced atoms as a function of depth. Two regions of disorder, one corresponding to the electronic damage at the surface and the other one corresponding to the nuclear damage inside the sample, are clearly observed. The thickness of the surface layer steadily increases with fluence as expected from damage accumulation and the consequent lowering of the effective threshold for amorphization. One may, indeed, assure that the damage is essentially due to electronic excitation since in that region $S_{e} \gg S_{n}$. The position of the inner damage peak coincides with the end of range of the ions. The same information has been obtained for all other cases listed in Table I.

The evolution of damage at the sample surface is particularly reliable (the calculation at the surface is independent of the dechanneling model even if the surface peak has to be taken into account, especially at low damage levels), and it is illustrated in Fig. 3 for all investigated cases. Experimental yield points can be reasonably fitted with a typical Avrami's curve (just intended as a mathematical tool),

$$
s=1-\exp \left[\left(-\phi / \phi_{0}\right)^{n}\right]
$$

in accordance with previous experiments. ${ }^{6,12}$ The two relevant parameters $\phi_{0}$ and $n$ obtained from the fit are listed in Table II. From Fig. 2 and Avrami's fits it is clear, then, that the observed damage is a strongly nonlinear function of fluence as expected from the thresholding characteristics of the electronic damage. The exponent $n$ measures the rate of disordering, $(d s / d \phi)_{\phi=\phi_{0}}=n / e \phi_{0}$, at the critical fluence $\phi_{0}$ and represents the nonlinearity of the damage response. On the other hand, $\phi_{0}$ stands for the fluence required to reach a critical level of disorder causing $\sim 63 \%$ of the full random (amorphous) RBS yield. Due to the sharpness of the curve, it does not substantially differ from the values corresponding to other choices of such critical disorder in the range of 0.5-0.9. In fact, the values for $n$ and $\phi_{0}$ (see Table II) show a large dispersion due to experimental errors associated with the abrupt character of the kinetics. Note that all curves show the same basic kinetic behavior. There is an initial region of fluence where no significant disorder is induced (incubation stage), so that point defects should be mostly created. From thereon lattice amorphization abruptly develops, coinciding with the abrupt rise of Avrami's curves. The incubation fluence (e.g., $\phi_{0}$ ) increases from $\mathrm{Cl} 45 \mathrm{MeV}$ to $\mathrm{O} 5 \mathrm{MeV}$ in order of decreasing electronic stopping power. It should be related to the fluence needed to accumulate defects and reach the threshold concentration causing amorphization. On the other hand, the lowest values corresponding to the highest $S_{e}$ become around $(3-5) \times 10^{12} \mathrm{~cm}^{-2}$ that may be related to the fluence required to amorphize the whole crystal surface and constitute a homogeneous layer.

One can now plot the dependence of such incubation fluence with electronic stopping power that is displayed in Fig. 4. For convenience we have defined such fluence as that one causing $50 \%$ amorphization (random RBS/C). It shows a

TABLE II. Parameters $\left(\phi_{0}\right.$ and $\left.n\right)$ derived from Avrami's fit to the damage kinetics together with the corresponding $\chi^{2}$ value. The electronic stopping power and nuclear scattering cross section at the target surface and the ion ranges are also included.

\begin{tabular}{cccccccc}
\hline \hline $\begin{array}{c}\text { Ion } \\
\text { energy } \\
(\mathrm{MeV})\end{array}$ & $\begin{array}{c}S_{e} \\
(\mathrm{keV} / \mathrm{nm})\end{array}$ & $\begin{array}{c}S_{n} \\
(\mathrm{keV} / \mathrm{nm})\end{array}$ & $\begin{array}{c}\phi_{0} \\
\left(10^{12} \mathrm{at.} / \mathrm{cm}^{2}\right)\end{array}$ & $n$ & $\chi^{2}$ & $\begin{array}{c}\sigma_{n} \\
\left(10^{-16} \mathrm{~cm}^{2}\right)\end{array}$ & $\begin{array}{c}\text { Ion } \\
\text { range } \\
(\mu \mathrm{m})\end{array}$ \\
\hline O 5.0 & 3.1 & 0.0023 & 400 & 2.5 & 0.0008 & 0.12 & 2.44 \\
F 5.1 & 3.4 & 0.0033 & 49 & 4 & 0.00456 & 0.17 & 2.50 \\
Si 5.0 & 4.2 & 0.010 & 11.2 & 2.2 & 0.00119 & 0.46 & 2.06 \\
Si 7.5 & 5.3 & 0.0082 & 5.5 & 4.2 & 0.00067 & 0.4 & 2.42 \\
Si 41 & 5.2 & 0.0027 & 18.2 & 3.6 & 0.00196 & 0.09 & 8.67 \\
Cl 11 & 6.1 & 0.0131 & 4.8 & 2.1 & 0.00102 & 0.44 & 3.09 \\
Cl 46 & 6.8 & 0.0025 & 5.1 & 2.2 & 0.00281 & 0.14 & 8.11 \\
\hline \hline
\end{tabular}




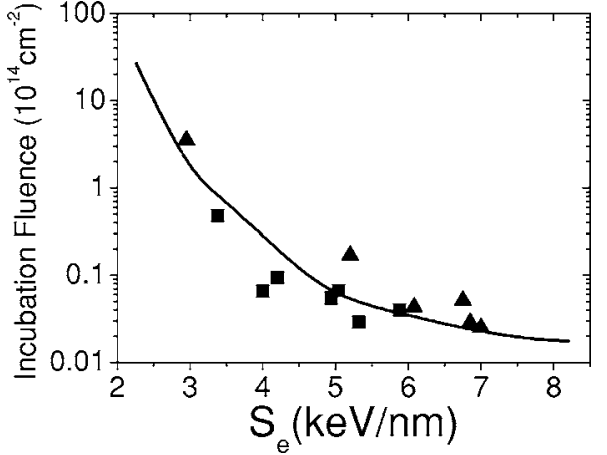

FIG. 4. Experimental data points for the incubation fluence $\phi_{0}$ vs stopping power (electronic) obtained from the measurements detailed in Table I and from Ref. 6. Squares have been used for ions with velocities lower than $0.3 \mathrm{MeV} / \mathrm{amu}$ and triangles for velocities greater than $0.3 \mathrm{MeV} / \mathrm{amu}$. The theoretical (continuous) curve stands for the Monte Carlo simulation using the following parameter values: $S_{\mathrm{th}}=4.4 \mathrm{keV} / \mathrm{nm}, a_{0}=3.18 \mathrm{~nm}$, and $k T_{m} / \varepsilon$ $=0.12$. Both experiment and theory correspond to $50 \%$ amorphization.

strongly nonlinear monotonic dependence on electronic stopping power, i.e., on the type of ion and energy.

In Avrami's curves used to describe the data, the initial rate of disorder is zero. However, this is not physically acceptable. What one can say instead is that such initial rate is low and cannot be reliably determined from the data. We will see later that it may be inferred from Avrami's parameters when one uses an adequate theoretical framework (see Secs. V-VII).

\section{GENERAL PHENOMENOLOGICAL DESCRIPTION}

In order to analyze the average damage kinetics represented by curves in Fig. 3, one may start by using a general phenomenological equation to describe the accumulation of average volume defect concentration $c$ of (point) defects created under irradiation,

$$
d c / d \phi=\left(1-c_{r}\right) g\left(S_{e}, S_{n}, c\right)
$$

where $c_{r}=c / N_{0}$ is the relative defect concentration in relation to the total concentration $N_{0}$ of available defect sites or sources. In principle, assuming homogeneous nucleation of defects, $N_{0}$ stands for the atomic density of the material. The equation includes the defect generation rate $g$ per unit depth [dependent on stopping powers and a factor $\left(1-c_{r}\right)$ describing the exhaustion of defect sources]. The generation rate can be alternatively described as $g=\sigma N_{0}, \sigma$ being the cross section for defect creation. The dependence of $g$ on $c_{r}$ might include coupling processes among damaged regions as well as possible indirect (defect-assisted) generation mechanisms.

In order to compare the predictions of Eq. (2) with $\mathrm{RBS} / \mathrm{C}$ data, one has to connect the defect concentration $c_{r}$ to the relative displaced fraction $s$ through a RBS/C defect scattering function, $s\left(c_{r}\right)$, that may depend on the specific defect structure. Recent calculations ${ }^{21}$ have provided the shape of this function for several defect structures in silicon. In the case of $\mathrm{LiNbO}_{3}$, such information is not available and, therefore, one is forced to make some convenient approximations on the function $s\left(c_{r}\right)$ to perform a meaningful analysis of the data and particularly learn about the transition from the isolated defect (preamorphous) region to the amorphous region.

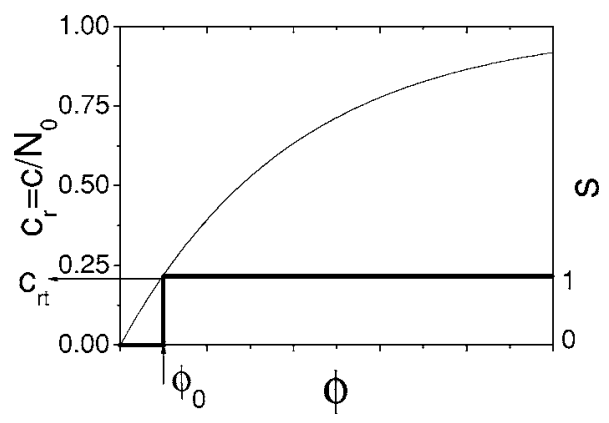

FIG. 5. Illustration of the kinetics for the relative defect concentration $c_{r}$ and displacements fraction $s$ as a function of fluence. $c_{\mathrm{rt}}$ corresponds to the critical concentration causing lattice amorphization.

At least one or both of the $g\left(S_{e}\right)$ and $s\left(c_{r}\right)$ functions have to be strongly nonlinear to account for the thresholding behavior of electronic damage. In principle, one may consider that the cumulative character of the damage together with the thresholding behavior provide the necessary physical bases to describe the disorder kinetics without invoking additional indirect processes. This assumption should be confirmed after comparing to experiment.

\section{THEORETICAL MODELING OF THE KINETICS: ANALYTICAL APPROACH}

Based on previous experimental evidence on single track as well as on high-fluence experiments, one may assume two different stages of structural damage. In a first stage point defects are generated whose concentration should be a smooth function of fluence. When a relative critical or threshold concentration $c_{\mathrm{rt}} \ll 1$ is reached, a structural change sets in and an amorphous phase is formed. In principle, base on the evidence available for high-energy medium-mass (swift) ions, one takes the growth rate $g$ to be exclusively a function of the electronic stopping power $S_{e}$ and so, for given irradiation conditions, it is constant at a fixed depth inside the crystal. Then, the equation describing the evolution of $c_{r}$ in an average analytical approximation (ignoring the statistics of ion impacts) is

$$
d c / d \phi=\left(1-c_{r}\right) g \text {. }
$$

The solution, illustrated in Fig. 5, is

$$
c_{r}=1-\exp \left\{-g \phi / N_{0}\right\} .
$$

On the other hand, the evidence in single track, ${ }^{3-6}$ as well as full layer ${ }^{12-16}$ experiments, suggests that $s\left(c_{r}\right)$ is a sharp function around the relative threshold concentration $c_{\mathrm{rt}}$ causing lattice collapse. Therefore, as a convenient limit, one may start by using a steplike function at $c_{\mathrm{rt}}$ and write

$$
\begin{aligned}
& s=0 \text { for } c_{r} \leqslant c_{\mathrm{rt}}<1, \\
& s=1 \text { for } c_{r}>c_{\mathrm{rt}}<1 .
\end{aligned}
$$

The combination of the two functions (4) and (5) provides an explanation for the thresholding behavior in the damage kinetics. Specifically, the model assumes that the displaced fraction $s(\varphi)$ would be a step function, as schematically illustrated in Fig. 5. Under the assumption $c_{\mathrm{rt}} \ll 1$, the fluence 
$\phi_{0}$ at which the step in $s$ occurs (i.e., the incubation fluence) would be given by

$$
\phi_{0} g=N_{0} c_{\mathrm{rt}}=c_{t} .
$$

Obviously, such step function only provides a rough approximation to the Avrami kinetics. However, one may identify $\phi_{0}$ with the fluence at which the areal fraction that is amorphized in Avrami's curves corresponds to $s=0.5$. In fact, due to the sharpness of the curves, the results do not depend critically on this particular choice for $s$. From $\phi_{0}$ one can then obtain the defect generation rate, $g$, in normalized units, through the simple equation

$$
g_{r}=g / c_{t}=1 / \phi_{0} .
$$

Using this expression, one may obtain a rough estimate of $g$ and consequently $\sigma$. As an example, assuming $c_{\mathrm{rt}}=0.1$, it comes out $\sigma=2 \times 10^{-14} \mathrm{~cm}^{2}$ for $\mathrm{Cl}$ at $46 \mathrm{MeV}$ and $\sigma=2$ $\times 10^{-15} \mathrm{~cm}^{2}$ for $\mathrm{F}$ at $5.1 \mathrm{MeV}$. One should note that these values are much higher than those corresponding to a nuclear collision mechanism (see Table II).

\section{DEFECT GENERATION RATES}

In view of the above analysis, it is now appropriate to calculate the defect generation rates in terms of a recent theoretical model ${ }^{17}$ based on the thermal spike concept and on the cumulative character of the damage. The model assumes that the point-defect concentration generated at a given spot is exclusively determined by the deposited energy $S_{e}$ and corresponds to thermal equilibrium at the maximum temperature $T(r)$ locally reached in the spike, $r$ being the distance to the ion trajectory (track axis). In accordance with thermodynamical arguments, the local defect concentration derives from a Arrhenius dependence

$$
c(r)=A \exp [-\varepsilon / k T(r)],
$$

with the activation energy $\varepsilon$ corresponding to the formation enthalpy of intrinsic defects. That concentration is assumed to be frozen during the fast cooling following the passage of the bombarding ion. Assuming Gaussian temperature profiles $^{7,18}$ one can write

$$
\begin{aligned}
& T\left(r, S_{e}\right)=\frac{\eta S_{e}}{\pi a_{0}^{2} \rho C} \exp \left(-r^{2} / a_{0}^{2}\right)+T_{S}, \quad 0<r<a_{0}, \\
& T\left(r, S_{e}\right)=\frac{\eta S_{e}}{\pi e \rho C} \frac{1}{r^{2}}+T_{S}, \quad r>a_{0},
\end{aligned}
$$

the meaning of the parameters being as follows: $a_{0}$ stands for the width of the Gaussian profile, $\eta$ is an efficiency factor that measures the fraction of deposited electronic energy transferred to the lattice as heat, $\rho$ is the material density, $C$ the specific heat, and $T_{s}$ the substrate temperature during irradiation. Combining (8) and (9), one obtains after some algebra ${ }^{17}$ that the linear density of defects $g$ produced by every single ion impact is

$$
\begin{aligned}
g\left(S_{e}\right) / c_{t}=\frac{g\left(S_{e}\right)}{N_{0} c_{\mathrm{rt}}} & =2 \pi \int_{0}^{\infty} r\left[c_{r}\left(S_{e}\right) / c_{\mathrm{rt}}\right] d r \\
& =2 \pi \int_{0}^{\infty} r \exp \left[-\varepsilon / k T\left(r, S_{e}\right)\right] d r
\end{aligned}
$$

that permits the numerical calculation of the relative generation rate of defects in terms of the relevant physical parameters of the model $\left(\varepsilon, a_{0}\right.$, and $\left.S_{\mathrm{th}}\right)$. Note that knowledge of $c_{\mathrm{rt}}$ is not required.

In order to calculate the defect concentration corresponding to a given fluence, one should add all single-impact contributions. However, this could only be done for very low enough fluences where single tracks are isolated (noninteracting). When track coupling occurs, the simple addition rule is not applicable. Since this is, in fact, the region of interest for our work, one should develop a strategy that takes into account track overlapping.

\section{MONTE CARLO APPROACH}

In order to properly describe the growth of defect concentration in the region where single damage tracks are interacting and before a homogeneous defective layer is formed, a Monte Carlo simulation method has been developed. ${ }^{18}$ It uses the physical model of Sec. VI but takes into account the statistics of impacts. Leaving aside technical details the method proceeds as follows: each bombarding ion impacts on an arbitrary point of the surface described by random coordinates. The generated temperature profile (spike) is calculated by (9) and then the local defect concentration at a given point is derived from (8). This concentration is added to the value generated by previous impacts and is stored to continue the process. In this way a map of defect concentrations is built. From that map the fractional area where the concentration has reached the critical value for amorphization is calculated as a function of increasing fluence. The curves obtained from such Monte Carlo approach for all ions investigated in this work are shown together with the experimental data in Fig. 3. The set of physical parameters used is $S_{\mathrm{th}}=4.4 \mathrm{keV} / \mathrm{nm}, a_{0}=3.18 \mathrm{~nm}, k T_{m} / \varepsilon=0.12$ (where $T_{m}$ is the melting temperature of $\mathrm{LiNbO}_{3}$ and $k$ is the Bolztmann constant). The abrupt shape of the amorphization curves is well predicted in rough accordance with Avrami's kinetics. Moreover, the general trend observed on changing ion and energy is also reasonably explained. However, the detailed quantitative agreement with experiment is not very good. This may be partly due to the large experimental errors that are expected in the irradiation experiments and in the analysis of the RBS/channeling data. Anyhow, the quality of the accordance between theory and experiment can be better appreciated by comparing the values for the incubation fluence. This is a more representative physical parameter and is free of some of the accidental errors associated with the detailed kinetic data. This comparison is illustrated in Fig. 4, where both data and simulation values are taken at 50\% areal fraction of full disorder. One can see that the agreement is, indeed, reasonable. An even more physically meaningful comparison can be made in reference to Fig. 6, where the 


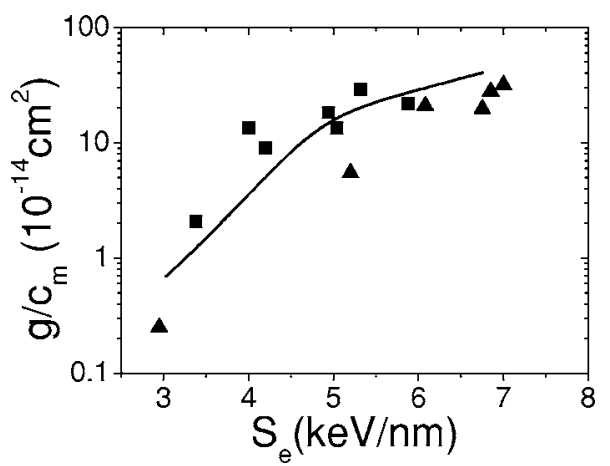

FIG. 6. Plot of the relative defect generation efficiency, $g / c_{m}$, vs the electronic stopping power $S_{e}$. Experimental points are obtained from Avrami's fit of the measurements detailed in Table I and in the Ref. 6. Squares have been used for ions with velocities lower than $0.3 \mathrm{MeV} / \mathrm{amu}$ and triangles for velocities greater than $0.3 \mathrm{MeV} / \mathrm{amu}$. The theoretical curve (continuous line) represents the result of the Monte Carlo simulations.

reciprocal of the incubation fluence (or relative efficiency for defect creation) is plotted versus the electronic stopping power. The theoretical Monte Carlo curve included in the plot shows again a reasonable agreement with the experimental behavior. However, one should note that experimental values are slightly displaced along the vertical axis depending on the ion velocity expressed in MeV/amu. Although data are not unambiguous, this may be an indication of the role of ion velocity on the damage efficiency ${ }^{6,10}$ (velocity effect).

\section{SUMMARY AND CONCLUSIONS}

First, the disorder and amorphization generated at a surface layer (far from the end of range depth) by a diversity of ions and energies clearly confirm that electronic excitation by the bombarding ions is the responsible mechanism. Moreover, the data reported in this paper provide a reasonable physical picture for the kinetics of damage and amorphization in $\mathrm{LiNbO}_{3}$. The process includes an incubation fluence where only pointlike defects are formed and the lattice disorder is not significant. At a certain critical fluence the disorder abruptly rises and leads to a transition to a full amorphous phase. The abrupt shapes of the kinetic curves can be simulated with a Monte Carlo method based on a thermal spike model and an Arrhenius law for the generation of intrinsic defects. In particular, the theory provides a reasonable quantitative agreement for the prediction of the incubation fluence and its systematic dependence on the electronic stop- ping power. Although the scheme presented here may need some refinements, as some coupling between electronic and nuclear processes and ion-velocity effects, we consider that it constitutes a good starting point for future developments.

\section{ACKNOWLEDGMENTS}

One of the authors (A.G.-N.) acknowledges the financial support of the MEC through a FPU Fellowship and of the Madrid City Hall-Residencia de Estudiantes

${ }^{1}$ Ion Implantation-2000 Science and Technology, edited by J. F. Ziegler (Lattice, Sunset Beach, CA, 2000).

${ }^{2}$ P. D. Townsend, P. J. Chandler, and L. Zhang, Optical Effects of Ion Implantation (Cambridge University Press, Cambridge, 1994).

${ }^{3} \mathrm{R}$. Spohr, in Ion Tracks and Microtechnology: Basic Principles and Applications, edited by K. Bethge (Vieweg, Braunchsweig, 1990).

${ }^{4}$ M. Toulemonde, S. Bouffard, and F. Studer, Nucl. Instrum. Methods Phys. Res. B 91, 108 (1994).

${ }^{5}$ B. Canut, S. M. S. Ramos, R. Brenier, P. Thevenard, L. J. Loubet, and M. Toulemonde, Nucl. Instrum. Methods Phys. Res. B 107, 194 (1996).

${ }^{6}$ S. M. S. Ramos, B. Canut, M. Ambri, N. Bonardi, M. Pitaval, H. Bernas, and J. Chaumont, Radiat. Eff. Defects Solids 143, 299 (1998).

${ }^{7}$ G. Szenes, Phys. Rev. B 51, 8026 (1995).

${ }^{8}$ M. Toulemonde, C. Trautmann, E. Balanzat, K. Hjort, and A. Weidinger, Nucl. Instrum. Methods Phys. Res. B 216, 1 (2004).

${ }^{9}$ A. Meftah, F. Brisard, J. M. Constantini, M. Hage-Ali, J. P. Soquert, F. Studer, and M. Toulemonde, Phys. Rev. B 48, 920 (1993).

${ }^{10}$ A. Meftah, J. M. Constantini, N. Khalfaoui, S. Boudjadar, J. P. Soquert, F. Studer, and M. Toulemonde, Nucl. Instrum. Methods Phys. Res. B 237, 563 (2005).

${ }^{11}$ G. G. Bentini et al., J. Appl. Phys. 92, 6477 (2002).

${ }^{12}$ G. G. Bentini et al., J. Appl. Phys. 96, 242 (2004).

${ }^{13}$ J. Olivares, G. García, F. Agulló-López, F. Agulló-Rueda, J. C. Soares, and A. Kling, Nucl. Instrum. Methods Phys. Res. B 242, 534 (2005).

${ }^{14}$ M. Bianconi et al., Appl. Phys. Lett. 87, 072901 (2005).

${ }^{15}$ J. Olivares, G. García, F. Agulló-López, F. Agulló-Rueda, A. Kling, and J. C. Soares, Appl. Phys. A: Mater. Sci. Process. 81, 1465 (2005).

${ }^{16}$ J. Olivares, G. García, A. García-Navarro, F. Agulló-López, O. Caballero, and A. García-Cabañes, Appl. Phys. Lett. 86, 183501 (2005).

${ }^{17}$ F. Agulló-López, G. García, and J. Olivares, J. Appl. Phys. 97, 093514 (2005).

${ }^{18}$ G. García, F. Agulló-López, J. Olivares, and A. García-Navarro, J. Appl. Phys. 99, 1 (2006).

${ }^{19}$ Properties of Lithium Niobate, EMIS Datareview Series, edited by K. K. Wong (INSPEC, Exeter, 2002).

${ }^{20}$ F. Agulló-López, J. M. Cabrera, and F. Agulló-Rueda, Electrooptics: Phenomena, Materials and Applications (Academic, London, 1994).

${ }^{21}$ M. Bianconi, E. Albertazzi, S. Balboni, L. Colombo, G. Lulli, and A. Satta, Nucl. Instrum. Methods Phys. Res. B 230, 185 (2005).

${ }^{22}$ The Stopping and Ranges of Ions in Solids, edited by J. F. Ziegler, J. P. Biersack, and U. Littmark (Pergamon, New York, 1985); see also the SRIM web page http://www.srim.org

${ }^{23}$ E. Albertazzi, M. Bianconi, G. Lulli, R. Nipoti, and M. Cantiano, Nucl. Instrum. Methods Phys. Res. B 118, 128 (1996). 